\title{
Cardiovascular Response to Defecating Postures with and without Valsalva Maneuver in Healthy Individuals
}

\author{
${ }^{1}$ Priyanka D Bhagat, ${ }^{2}$ Mariya P Jiandani, ${ }^{3}$ Amita A Mehta
}

\begin{abstract}
Aims: To assess the cardiovascular response to defecating postures of bridging and sitting lean forward with and without Valsalva maneuver in healthy individuals.
\end{abstract}

Study design: Prospective interventional cross-over study in healthy population.

Study material: Stop watch, Rubber tube, Nostril clip, Pillow, Mercury Sphygmomanometer, Cardioscope (Model no.: Iris 50, Maestros company).

Materials and methods: A total of 100 healthy individuals participated in the study. They maintained sitting lean forward and in bridging position for 5 minutes with and without Valsalva maneuver. Valsalva maneuver was performed at $40 \mathrm{~mm} \mathrm{Hg}$ pressure for 15 seconds. Blood pressure and heart rate were recorded. Mean arterial pressure and rate pressure product were derived.

Data analysis: The data was analyzed using Statistical Package for the Social Sciences v 16. Shapiro-Wilk test was used to test normality, and nonparametric Mann-Whitney test was used with $p<0.05$ as statistically significant.

Results: Sitting in lean forward position showed statistical significant increase in heart rate $(p<0.05)$ compared to bridging position with Valsalva maneuver. There was a significant rise in blood pressure with bridging as compared to sitting lean forward with Valsalva. There was no statistically significant difference in heart rate and blood pressure between sitting lean forward position and bridging position without Valsalva maneuver.

Conclusion: Sitting lean forward position and bridging position increase hemodynamic load compared to relaxed sitting posture. With Valsalva maneuver sitting lean forward posture causes greater rise in heart rate compared to bridging position.

Keywords: Bridging position, Cardiovascular response, Sitting lean forward, Valsalva maneuver.

How to cite this article: Bhagat PD, Jiandani MP, Mehta AA. Cardiovascular Response to Defecating Postures with and without Valsalva Maneuver in Healthy Individuals. Int J Recent Surg Med Sci 2016;2(1):10-14.

Source of support: Nil

Conflict of interest: None

${ }^{1}$ Physiotherapist, ${ }^{2,3}$ Associate Professor

${ }^{1}$ Department of Physiotherapy, Seth GS Medical College \& King Edward Memorial Hospital, Mumbai, Maharashtra, India

2,3Department of Physiotherapy School and Centre, Seth GS Medical College \& King Edward Memorial Hospital, Mumbai Maharashtra, India

Corresponding Author: Priyanka D Bhagat, Physiotherapist Department of Physiotherapy, Seth GS Medical College \& King Edward Memorial Hospital, Mumbai, Maharashtra, India, Phone: +919860941609, e-mail: bhagatpriya15@gmail.com

\section{INTRODUCTION}

Posture is known to have important physiologic effects on the cardiovascular system at rest, during exercise, and activities of daily living (ADL). The distribution of blood and the hydrostatic forces acting between the cardiac pump and the peripheral vasculature are altered when a subject assumes different body posture. "Activities of Daily Living are a term used in health care to refer to everyday self-care activities." Ambulation, dressing, eating, bathing, defecation, and micturition are basic ADL. ${ }^{2}$ According to Japanese Society for Hygiene, 8 to $10 \%$ of cardiovascular arrest occurred in individuals while they were in the toilet. ${ }^{3}$ The mechanism responsible for the disproportionately high frequency of cardiac arrest associated with micturition and defecation was due to the sudden elevation of blood pressure and imbalance between sympathetic and parasympathetic nervous system. ${ }^{4}$

The Valsalva maneuver (VM) is classically defined as forcible exhalation against a closed glottis, which increases pressure within thoracic cavity and impedes venous return of blood to the heart. ${ }^{5}$ The extracardiac lesion, i.e., subarachnoid hemorrhage, aortic dissection, rupture of abdominal aortic dissection, pulmonary embolism, all these vascular diseases may trigger by sudden rise in blood pressure that occurs during the VM. ${ }^{6}$ There is a common agreement that VM has adverse effect on cardiovascular system and is one of the causative factors for defecation syncope and sudden death in elderly individuals. ${ }^{7-9}$ The VM associated with the act of defecation/micturition may be considered as one of the triggering factor for cardiovascular incidence in compromised cardiovascular disease population. ${ }^{10,11}$

Normal defecation consists of three components: Spontaneous phasic rectal contraction, relaxation of the anal canal, and straining (somatic component). Among these, straining is the most significant in the etiology of pathological conditions. ${ }^{12}$ The voluntary straining while the glottis is closed and increase in intra-abdominal and intra-thoracic pressure are the chain of events that are associated with VM. Generating high intra-abdominal pressure and frequent release of "breath holding" has great impact on hemodynamic status. ${ }^{13}$

There are four physiological phases of VM: (i) Onset of strain; (ii) continued strain; (iii) release; and (iv) recovery. Phase I: Exhaling against an obstructed airway (closed 
glottis) causes increased intra-thoracic pressure and a rise in systolic blood pressure (SBP) due to compression of the aorta. Phase II: This is followed by a decrease in venous return and a decline in SBP to below baseline as a positive intra-thoracic pressure is maintained. The release and recovery phases represent compensatory phases for the normal physiologic mechanisms which are activated after decline in intra-thoracic pressure, i.e., in phase III and IV. In phase III compensation leads to decrease in SBP caused by initial pooling of blood in the pulmonary vasculature and phase IV shows overshoot in SBP because of sudden increase of cardiac output and vasoconstriction from sympathetic over activity.

Many previous studies have evaluated cardiovascular response in different stress provoking defecation positions, such as squatting by performing VM in normal individuals. But the impact of sitting lean forward (SLF) and bridging (BRG) positions, which are the most common acquired defecation positions in acute care, has not been evaluated. Hence, this study aims to evaluate the cardiovascular response in SLF and BRG positions with and without VM in healthy individuals.

\section{MATERIALS AND METHODS}

It was an observational cross-over study. Approval from Institutional Ethics Committee and State University was obtained. The study was conducted at the Physiotherapy Center of a multispecialty tertiary care hospital. A total of 100 Healthy individuals of age 30 to 60 years and of either sex who gave written consent were randomly selected. Subjects were excluded from the study if they were unable to comprehend the instruction or if they had poor effort to perform VM. Relaxed sitting position (Fig. 1) was maintained as starting position and baseline parameters, i.e., heart rate and blood pressure were recorded on cardioscope. The subjects then adopted either SLF (Figs 2A and B) or BRG (Figs 3A and B) posture first and

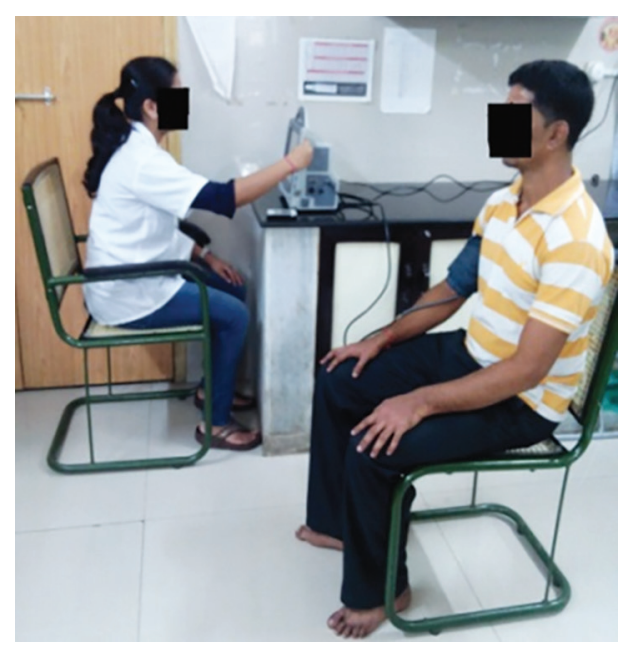

Fig. 1: Relaxed sitting position

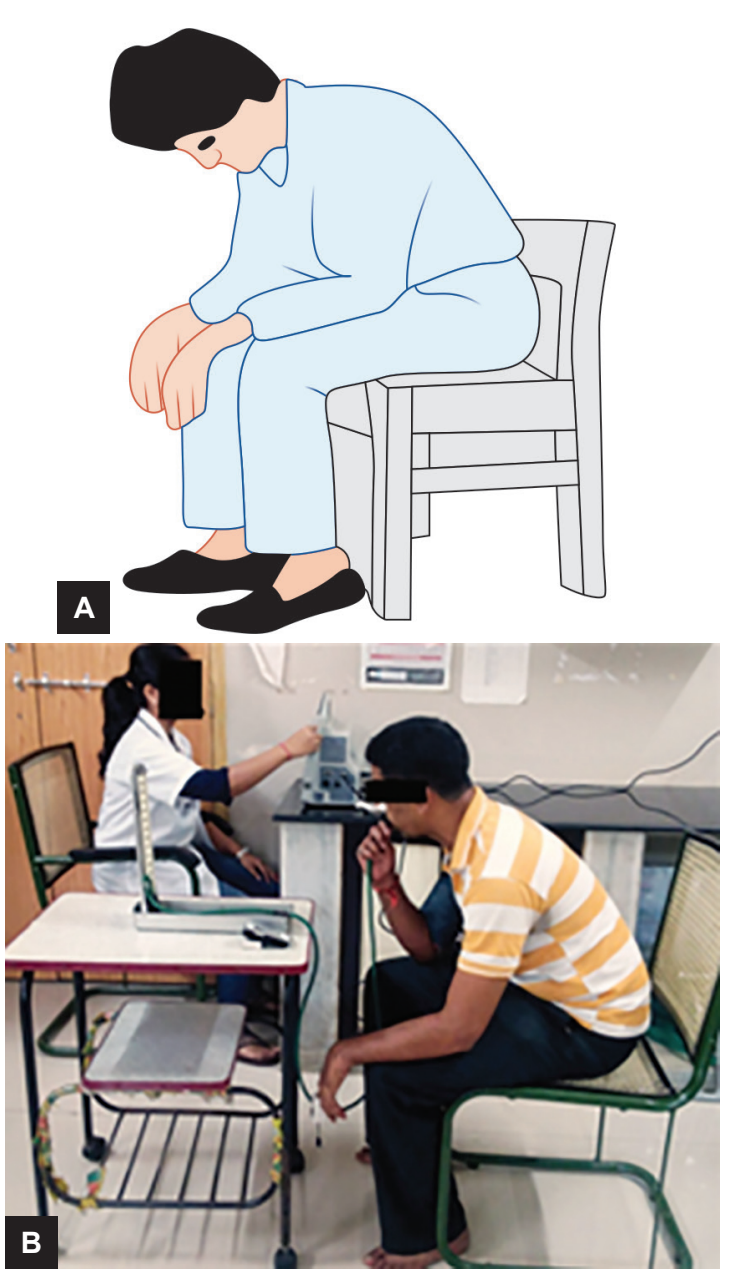

Figs $2 A$ and $B$ : (A) Sitting lean forward without $V M$, and (B) Sitting lean forward with VM
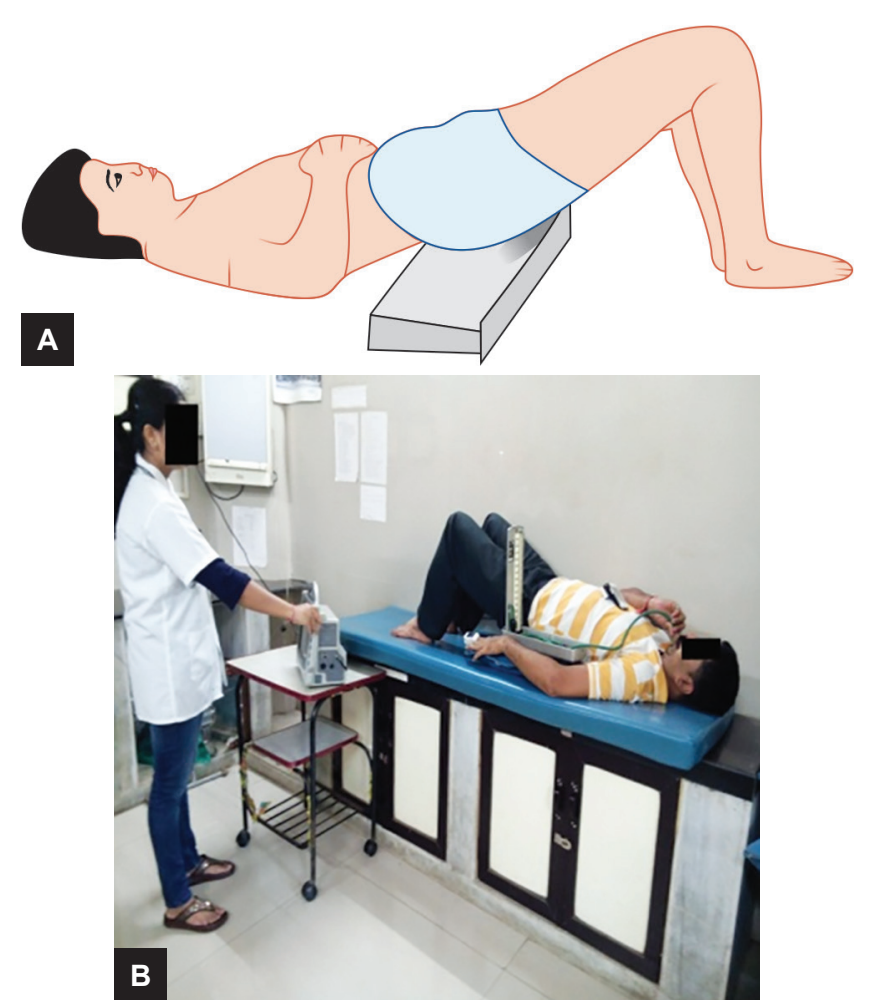

Figs $3 A$ and $B:(A)$ Bridging without $V M$, and $(B)$ Bridging with VM 
maintained it for 5 minutes, following which parameters were recorded again as changes due to adapted position (without Valsalva). The subjects were then allowed to rest before resuming back to SLF and BRG positions to be performed with a VM. Immediately post VM parameters were recorded with the help of cardioscope at the end of 5 minutes.

Valsalva was performed by raising the mercury column from 35 to $40 \mathrm{~mm} \mathrm{Hg}$ for 15 to 20 seconds into the mouthpiece that was attached to a sphygmomanometer. A small needle is inserted into the rubber tube attached to the sphygmomanometer so that the glottis remained open for the measurement of thorax pressure into the sphygmomanometer. As there was a postural modification to perform standardized VM procedure by maintaining airway pressure at 35 to $40 \mathrm{~mm} \mathrm{Hg}$ for 15 to 20 seconds, it is called as modified Valsalva maneuver (MVM). Mean arterial pressure (MAP) and rate pressure product (RPP) were derived from recorded blood pressure and HR using the formula: $(\mathrm{MAP})=[(2 \times \mathrm{DBP})+\mathrm{SBP}] / 3 ;(\mathrm{RPP})=\mathrm{HR} \times \mathrm{SBP}$.

\section{RESULTS}

The mean age of the individuals was $42.6 \pm 9.1$. There were $74 \%$ males and $26 \%$ females. The mean resting heart rate $(\mathrm{HR})$ was $79.22( \pm 6.2) \mathrm{bpm}$ and resting systolic and diastolic BP were $124( \pm 4.9)$ and $84.6( \pm 6.0) \mathrm{mm} \mathrm{Hg}$

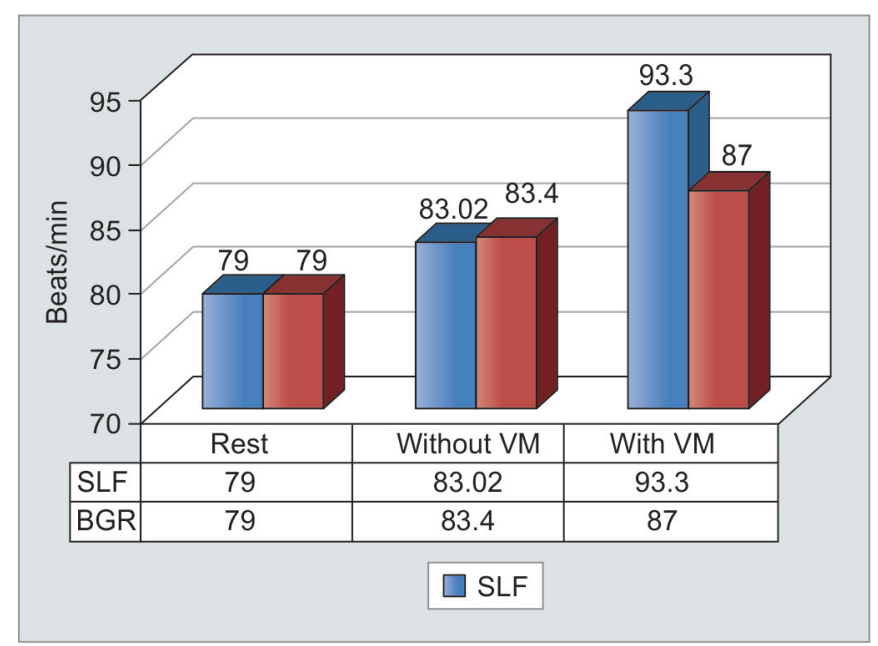

Graph 1: Change in mean HR in different positions with and without Valsalva

Impression: The mean value of HR shows statistically significant between rest and SLF and BRG positions; Within Group Analysis for HR: Sitting lean forward shows statistically significant increase in HR with VM compared to without VM, whereas BRG shows no statistical significant difference; and Inter-group comparison between SLF and BRG: No statistical significant difference of HR between SLF and BRG positions without VM $\left(p<0.05^{*}\right)$. Whereas, HR increased statistically with VM in SLF compared to $B R G$ position respectively. Normality was assessed using the ShapiroWilk test. The data did not follow normal distribution; therefore nonparametric test was used for statistical analysis with $\mathrm{p}<0.05$ as significant. Intra-group analyses of mean of variables were carried out using Friedman test. Inter-group analyses of mean of variables were done using Mann-Whitney U test.

There was a significant rise $(\mathrm{p}<0.05)$ in HR (Graph 1) and BP in both SLF (Graph 2) and BRG (Graph 3) posture compared to baseline resting position. However, there was no significant change in HR or BP between SLF and BRG postures without Valsalva. On comparison of SLF and BRG with MVM, there is a rise in HR with SLF posture $(\mathrm{p}<0.05)$ and rise in SBP $(\mathrm{p}<0.01)$ with BRG.

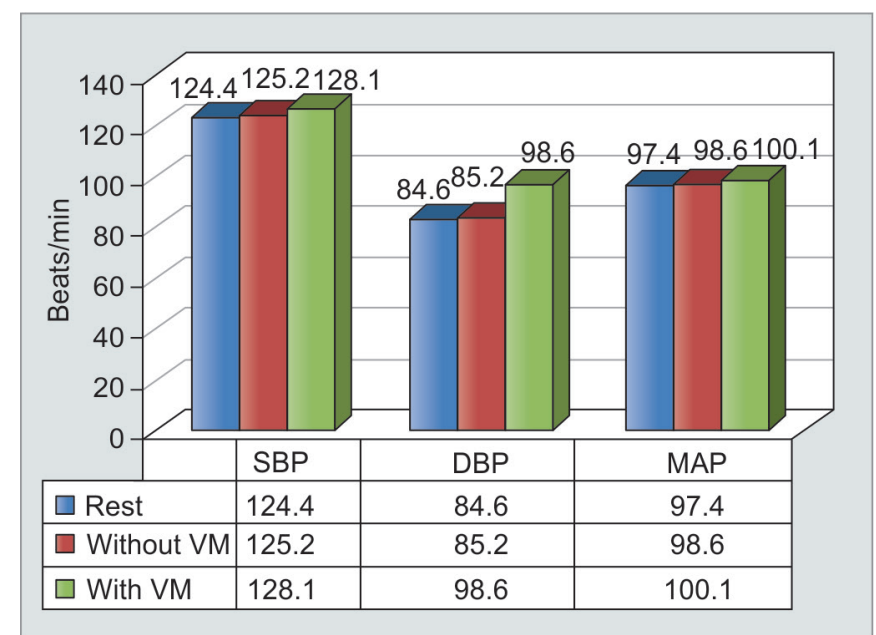

Graph 2: Mean value of pressure in SLF (with and without VM) Impression: Mean value of SBP and MAP shows highly statistical significant difference in SLF with VM as compared to rest and without VM $\left(p<0.001^{*}\right)$

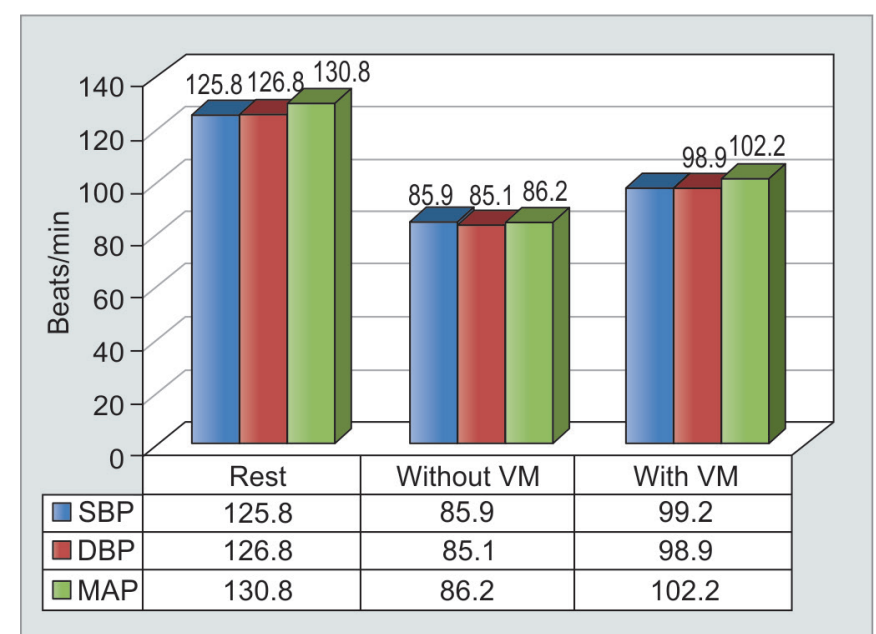

Graph 3: Mean value of pressure in BRG (with and without VM) Impression: Mean value of SBP and MAP shows statistical significant difference with VM as compared to rest and without VM and in between with and without VM $\left(p<0.01^{*}\right)$ 


\section{DISCUSSION}

In our study we found that there was a significant rise in heart rate and blood pressure with SLF and BRG postures compared to resting relaxed sitting posture. This is supported by Rathod et al study. ${ }^{14}$ In healthy adults, autonomic balance does not change significantly with different recumbent postures, ${ }^{15,16}$ but is clearly different between supine and vertical postures (standing or sitting). Sympathetic nervous function predominates in vertical postures, while vagal function predominates in recumbent postures. Diaphragmatic elevation and increased intra-thoracic and intra-abdominal pressure caused by SLF can have marked effect on cardiac contractility and HR. ${ }^{17}$ Arterial $\mathrm{BP}$ is a product of cardiac output (heart rate $\times$ stroke volume) and total peripheral resistance.

Shamsuzzaman et al has shown that antigravity muscle activity influences vasomotor and cardiovascular activity during postural change. It is likely that the sitting posture minimized antigravity muscle involvement and bridging enhanced it. On comparing SLF with BRG posture without VM, there is no statistically significant difference between the two positions. However, on addition of Valsalva, the HR increased significantly with SLF and SBP rose significantly with BRG posture. This was reflected in the derived variables of MAP and RPP.

Sitting lean forward compressed the abdominal contents and inferior vena cava reducing the preload which increases the HR to maintain the cardiac output. Bridging position resembles a supine position in which gravity is acting equally on the body surface. Supine position prevents adequate preload toward the heart and produces "flat-top" response. ${ }^{18}$ The flat-top response prevents development of early phase II of VM, i.e., drop in blood pressure due to venous and stroke volume reduction which mediates baroreflex vasoconstriction and increase in BP in Late phase II of VM. In bridging with elevation of the pelvic angle, the venous return is facilitated and this would increase the preload further and increase SBP (Graph 4). Also contraction of large mass of antigravity muscles would increase peripheral resistance,

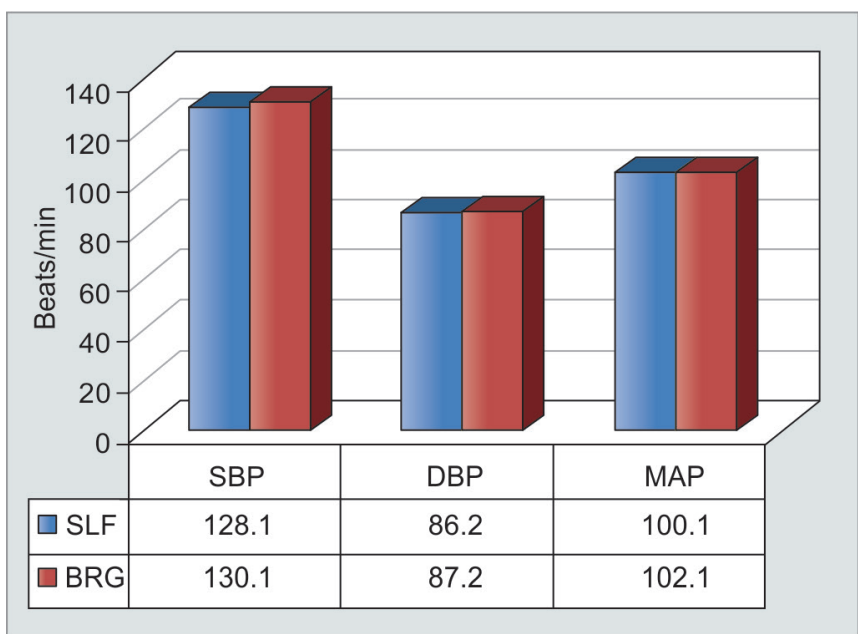

Graph 4: Mean value of BP between SLF and BRG with VM Impression: Mean value of SBP, MAP, and RPP shows higher statistical value in BRG compared to SLF with VM

hence diastolic blood pressure (DBP). Increasing intraabdominal and intra-thoracic pressure causes increase in myocardial oxygen consumption by increasing RPP.

The effect of VM in middle-aged individual poses little or no risk. The moderate change in position from flat to $60^{\circ}$ head up position does not affect the hemodynamic status of critically ill patients. ${ }^{19}$ However, in individuals suffering from coronary vascular diseases and frequent breath holding could be sufficient for the development of significant cardiac arrhythmia and sudden cardiac death. Thus patient with coronary vascular diseases on prolonged bed rest should be given stool softener routinely and instructed to breathe through the mouth when straining for defecation ${ }^{20}$ and during turning on bed or pulling themselves up in bed.

\section{CONCLUSION}

Sitting lean forward and bridging both result in a statistically significant increase in HR and blood pressure compared to relaxed sitting posture enhancing the hemodynamic load. On comparison of SLF and BRG position without VM (Table 1), no significant changes were observed in HR, SBP, DBP, MAP, and RPP. Whereas, with VM SLF shows significant changes in HR, SBP, MAP, and RPP as compared to BRG position.

Table 1: Comparison of mean scores of HR, SBP, DBP, RPP, and MAP with and without VM between SLF and BRG positions

\begin{tabular}{|c|c|c|c|c|c|c|}
\hline \multicolumn{4}{|c|}{ Without MVM } & \multicolumn{3}{|c|}{ With MVM } \\
\hline Mean/SD & $S L F$ & $B R G$ & $p$-value* & $S L F$ & $B R G$ & $p$-value* \\
\hline HR & $83.2( \pm 6.3)$ & $83.4( \pm 5.8)$ & $<0.98$ & $93.3( \pm 8.6)$ & $87.0( \pm 7.7)$ & $<0.05$ \\
\hline SBP & $125.2( \pm 5.4)$ & $126.6( \pm 6.1)$ & $<0.37$ & $128.1( \pm 6.4)$ & $130.8( \pm 6.8)$ & $<0.01^{*}$ \\
\hline DBP & $85.2( \pm 6.1)$ & $85.1( \pm 6.0)$ & $<0.06$ & $86.2( \pm 5.8)$ & $87.8( \pm 6.0)$ & $<0.08$ \\
\hline RPP & $10,375.24(879.5)$ & $10,508( \pm 933.3)$ & $<0.06$ & $11,951( \pm 1,228.9)$ & $11,310( \pm 1,332)$ & $<0.03$ \\
\hline MAP & $98.6( \pm 4.6)$ & $98.9( \pm 5.0)$ & 0.51 & $100.1( \pm 4.6)$ & $102.2( \pm 5.0)$ & $<0.05^{*}$ \\
\hline
\end{tabular}

*indicate significant values; Impression: Inter-group comparison between SLF and BRG without Valsalva shows no statistical significance. HR, BP, MAP, and RPP show statistical significance in SLF compared to BGR position 


\section{ACKNOWLEDGMENT}

Authors would like to thank Miss Namrata Navge for helping and drafting the research project.

\section{REFERENCES}

1. Moberg N. Hemodynamic Model of the cardiovascular system during the Valsalva Maneuver and orthostatic changes. UMEA University Master thesis in Engineering Physics SE-901 87 Umeå Sweden; 2011 Nov 23.

2. Pendleton HM. Activity of daily living. In: Pedretti's occupational therapy - Practice skill for physical dysfunction. Occupation performance and the performance areas: Evaluation and intervention. St Louis (MO): Elsevier (a division of Reed Elsevier India Private limited); 2006.

3. Inamasu J, Miyatake S. Cardiac arrest in the toilet: clinical characteristics and resuscitation profile. Jpn Soc Hygiene 2013 Mar;18:130-135.

4. Culić V. Triggering of cardiovascular incident by micturition and defecation. Int J Cardiol 2006 May;109(2):277-279.

5. Julian M, Marvin S, Medow A. Effect of thoracic blood volume on Valsalva maneuver. Am J Physiol Heart Circ Physiol 2004 Aug;287(2):H798-H804.

6. Inamasu J. Cardiac arrest in the toilet: clinical characteristic and resuscitation profiles. Environ Health Prev Med 2013 Mar;18:130-135.

7. Sikriov BA. Cardiovascular events at defecation: are they unavoidable? Med Hypotheses 1990 Jul;32(3):231-233.

8. Halpern A, Shaftel N, Selman D, Shaftel HE, Kuhn PH, Samuels SS, Birch HG. The straining forces of bowel function. Angiology 1960 Oct;11:426-436.
9. Matsuda M, Watanabe K, Saito A, Matsumura K, Ichikawa M. Circumstances, activity, and events precipitating, aneurysmal subarachnoid hemorrhage. J Stroke Cerebrovasc Dis 2007 Jan-Feb;16(1):25-29.

10. Kapoor WN, Peterson J, Karpf M. Defecation syncope. Arch Intern 1986 Dec;146:2377-2379.

11. Culić V. Triggering of cardiovascular incidents by micturition and defecation. Int J Cardiol 2006 May;109(2):277-279.

12. Sakakibara R. Influence of Body position on defecation in humans. LUTS 2010;2(1):16-21.

13. Priyanshu V, Rathod, et al. Cardiovascular responses with VM during activity of daily living in healthy adults. NJIRM 2010;1(2):6-11.

14. Rathod PPV, Ravindra PS, Nambiar PVK. Cardiovascular responses with valsalva maneuver during activities of daily livings in healthy adults. NJIRM 2010 [cited 2016 Apr 14]; 1(2):6-11.

15. Ryan AD, Larsen PD, Galletly DC. Comparison of heart rate variability in supine, and left and right lateral positions. Anaesthesia 2003 May;58(5):432-436.

16. Kalisnik JM, Avbelj V, Trobec R, Gersak B. Imaging of power spectral heart rate variability regarding subject position. Pflugers Arch 2001;442 6 (Suppl 1):R142-R144.

17. Elizabeth R. Effect of position on VM: Supine vs 20 degree position. J Clin Neurophysiol 2008 Oct;25(3):313-316.

18. Nobuhiro W. Effect of body position on autonomic regulation of cardiovascular functions in young, healthy adults. Chiropractic Osteopath 2007;15:19.

19. Zema MJ, Restivo B, Sos T, Sniderman KW, Kline S. Left ventricular dysfunction and bedside VM. Br Heart J 1980 Nov;44: 560-569.

20. MacWilliam JA. Postural effect on heart-rate and blood pressure. Exp Pyhsiol 1933 Aug;23(1):1-33. 